POS $\quad \begin{aligned} & \text { PROCEEDINGS } \\ & \text { OF SCIENCE }\end{aligned}$

\title{
Nuclear Physics from QCD
}

\section{U. van Kolck* ${ }^{\dagger}$}

Department of Physics, University of Arizona, Tucson, AZ 85721, USA

E-mail: vankolck@physics.arizona.edu

Effective field theories provide a bridge between QCD and nuclear physics. I discuss light nuclei from this perspective, emphasizing the role of fine-tuning.

8th Conference Quark Confinement and the Hadron Spectrum September 1-6 2008

Mainz, Germany

\footnotetext{
${ }^{*}$ Speaker.

${ }^{\dagger}$ Research supported by the U.S. Department of Energy.
} 


\section{Introduction}

Nuclear physics has long been recognized as rich and complex. The origin of this complexity has, however, been more diffi cult to pinpoint. About fi fteen years ago a road-map was traced [1] to link nuclear physics to QCD using effective fi eld theory (EFT). The idea is to break the problem into two steps: one is to calculate nuclear observables from a low-energy EFT; another is to obtain the EFT from QCD. The second step, where great progress has been achieved recently through lattice simulations, was addressed [2] by Paulo Bedaque at this conference. Here I focus on the first step in its simplest context, that of few-nucleon systems. Bigger nuclear systems were discussed [3] by Wolfram Weise. As we are going to see, complexity, at least in light-nuclear structure, is largely due to fi ne-tuning.

It is a common situation that we are interested in physics at a certain momentum scale $M_{l o}$, having only partial knowledge of the underlying theory characterized by a scale $M_{h i} \gg M_{l o}$. The crucial feature of EFT that sets it apart from models is that it incorporates a consequence of the marriage between quantum mechanics and relativity: everything that can happen will happen -at least virtually. Therefore, interactions among the low-energy degrees of freedom take all possible forms allowed by the symmetries of the underlying theory. In separating out some degrees of freedom as "low-energy", we introduce an arbitrary ultraviolet momentum cutoff $\Lambda$. Model independence requires that low-energy observables be independent of $\Lambda$-renormalization-group (RG) invariance. Facing an infi nite number of contributions to processes at momenta $Q \sim M_{l o}$, the only way to make predictions is to set up an expansion in powers of $Q / M_{h i}$, referred to as power counting. Truncating at any given order, we neglect contributions with higher powers of $Q / M_{h i}$ and $Q / \Lambda$, a breaking of RG invariance being unavoidable but suffi ciently small as long as $\Lambda \gtrsim M_{h i}$. Accuracy can be improved systematically by calculating higher-order contributions.

In the nuclear context, there are several relevant scales. First, QCD is characterized by an intrinsic scale $M_{Q C D} \sim 1 \mathrm{GeV}$, which sets the size of most hadron masses, for example the nucleon mass $m_{N} \simeq 940 \mathrm{MeV}$. The glaring exception is the pion, whose mass $m_{\pi} \simeq 140 \mathrm{MeV}$ signals its character as a pseudo-Goldstone boson. Pion interactions are characterized by the pion decay constant $f_{\pi} \simeq 93 \mathrm{MeV} \sim M_{Q C D} / 4 \pi$. Moreover, low-energy pions can excite a nucleon to a delta isobar, introducing the mass-splitting scale $m_{\Delta}-m_{N} \simeq 300 \mathrm{MeV}$. For simplicity I lump these three lower scales together into a common scale $M_{n u c} \sim 100 \mathrm{MeV}$. As we are going to see shortly, $M_{n u c}$ sets the scale of nuclear interactions - for example, the two-nucleon effective ranges $r_{N N} \sim 1 / M_{n u c}$. Still, there is a less obvious, smaller momentum scale: $\boldsymbol{N} \sim 1 / a_{N N} \sim 30 \mathrm{MeV}$, where $a_{N N}$ stands for some average of the two-nucleon scattering lengths. The appearance of this scale, which we will also discuss in the next section, determines much of the structure of light systems.

These multiple scales beg for multiple EFTs. Each EFT holds in a certain energy region; each, for particular values of its parameters, is QCD in that region. For other values of the parameters, these EFTs represent other possible underlying theories with the same symmetry pattern as QCD, but different dynamics. Because they carry less and less imprints of QCD - the RG is a semigroup, after all - the goal in building EFTs at lower and lower energies is not to test QCD, but to understand the emergence of nuclear structure from QCD. The three EFTs developed so far to deal with light nuclei are briefly discussed below. It is of course impossible to review fi fteen-plus years in twenty minutes. I will concentrate on the thread provided by fi ne-tuning. Other results and 
references can be found in various reviews $[4,5,6,7,8]$.

\section{Pionful EFT}

The first nuclear EFT to be considered [1] is also the closest to QCD: the generalization of chiral perturbation theory $(\chi \mathrm{PT})$ to systems with two or more nucleons. In this EFT, $M_{l o}=M_{n u c}$ and $M_{h i}=M_{Q C D}$ : the low-energy degrees of freedom are (non-relativistic) nucleons, pions, and (non-relativistic) delta isobars; the symmetries, Lorentz, approximate parity, time-reversal, and chiral. The Lagrangian is the one familiar in $\chi \mathrm{PT}$ applications to processes with one or no nucleon, supplemented by contact interactions among nucleons and deltas. (The same ideas can be applied [9] to other "nuclei", such as possible hidden-charm "molecules".)

Not everything is just a trivial extension, though. There are two related, new issues. First, contributions to nuclear amplitudes exist where intermediate states differ from initial states only in the kinetic energies of nucleons, which are of $\mathscr{O}\left(Q^{2} / M_{Q C D}\right)$-in contrast, in hadronic processes generic intermediate states have energies of $\mathscr{O}(Q)$. Contributions from unitarity cuts are thus infrared enhanced [1] and typically of $\mathscr{O}\left(M_{Q C D} Q / 4 \pi\right)$. Since one-pion exchange is of $\mathscr{O}\left(1 / M_{n u c}^{2}\right)$, ladder contributions need to be resummed in low partial waves - in the two-nucleon case, $l \leq 2$ [10]. (Inverse factors of angular momentum $l$ suppress loop contributions in high waves.) As a consequence, poles can appear in $T$ matrices for momenta $Q \sim 4 \pi M_{n и с}^{2} / M_{Q C D} \sim M_{\text {пис }} \sim 100 \mathrm{MeV}$, or equivalently for binding energies $B \sim Q^{2} / m_{N} \sim M_{n u c}^{2} / M_{Q C D} \sim 10 \mathrm{MeV}$. Nuclei thus arise naturally within EFT as a consequence of the existence of the scale $M_{n u c} \ll M_{Q C D}$ associated with pion physics.

The infrared-enhanced contributions come from states with no pions or deltas. Hence it is convenient to defi ne [1] a potential as the sum of Feynman diagrams that are nucleon irreducible. The estimate of the sizes of pion-exchange contributions to the potential is similar to that done in $\chi \mathrm{PT}$ with at most one nucleon, and the long-distance nuclear potential thus has [1] an expansion in $Q / M_{Q C D}$ from an increasing number of pion exchanges. The main pieces of the long-range potential have been calculated $[1,4,5]$ - for example, the two-pion-exchange three-nucleon potential is a modifi cation [11] of the popular Tucson-Melbourne potential - and incorporated in more traditional approaches, such as the Nijmegen phase-shift analysis [12], where they partially replace heavy-meson exchanges.

The second new issue is the size of fermion contact interactions. Until we have a solution for low-energy QCD, the best we can do is dimensional analysis constrained by RG invariance: one looks at the change in the contribution from an arbitrary loop under a natural variation in cutoff - that is, a variation by a factor of $\mathscr{O}(1)$, say 2 or $1 / 3$ - and demands that the size of the contact interactions of the same form be at least of the same size. In $\chi \mathrm{PT}$ with at most one nucleon, where all loops are (except in small windows of phase space) perturbative, the corresponding counterterms scale with inverse powers of $M_{Q C D}$ in a reasonably simple form, dubbed naive dimensional analysis [13]. For two or more nucleons there is (for $l \leq 2$ in two-body subsystems) an infi nite number of leading-order loops, encapsulated in a Schrödinger equation with the one-pion-exchange potential, which behaves as $1 / f_{\pi}^{2} r^{3}$ at short distance $r$. The non-perturbative renormalization of such singular potentials can also be dealt with by looking at natural cutoff variations in the Schrödinger equation. Surprisingly, in all waves where the singular potential is attractive, the counterterms depend on $\Lambda$ 

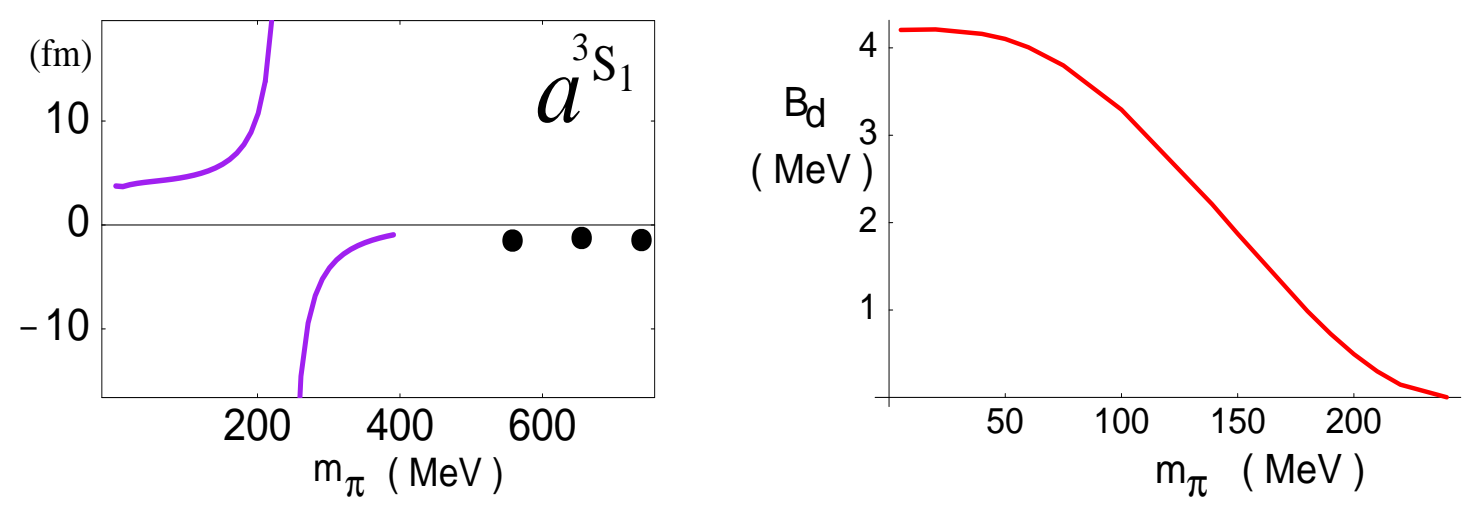

Figure 1: Dependence of the two-nucleon ${ }^{3} \mathrm{~S}_{1}$ scattering length $a^{3} S_{1}$ (left panel) and deuteron binding energy $B_{d}$ (right panel) on the pion mass $m_{\pi}$. The solid lines are results [17] from an incomplete sub-leading-order EFT calculation, where $m_{\pi}$ in the one-pion-exchange potential was varied. The dots are quenched lattice data [19].

in a limit-cycle-like fashion $[14,15]$, and their sizes are effectively driven by the scale appearing in the singular potential, rather than $M_{h i}$. In the nuclear case this scale is $f_{\pi}$, and thus certain contact interactions are larger $[16,17,10]$ than expected on the basis of naive dimensional analysis. Fortunately one can show [15] that the size of sub-leading contact interactions relative to the leading ones can still be estimated using naive dimensional analysis, and, together with the sub-leading pieces of the long-range potential, can be treated in perturbation theory in scattering amplitudes. The end result is that at each order RG invariance demands more contact interactions $[16,17,10]$ than assumed in earlier work. (For a dissenting view, see Ref. [18].)

Much attention has been dedicated to this EFT, although predominantly in its original version [1]: many encouraging results for scattering and bound states have been obtained $[4,5]$ and certainly many more are to come. Here I will mention only the appearance of fi ne-tuning.

In this EFT the pion mass can be varied, as long as $m_{\pi} \ll M_{Q C D}$. Thus EFT parameters can be fi tted to lattice results at suffi ciently small pion masses. The idea is illustrated in Fig. 1 , where the triplet two-nucleon scattering length $a_{N N}^{3} S_{1}$ and the deuteron binding energy $B_{d}$ in an incomplete sub-leading order [17] are shown together with old, quenched lattice data [19]. We can see that generically, as indicated by dimensional analysis, $a_{N N} \sim 1 / M_{n u c}$ and $B_{d} \sim M_{n u c}^{2} / M_{Q C D}$. However, a larger scattering length, or equivalently a smaller deuteron binding energy, arises from the seemingly accidental proximity between the observed $m_{\pi}$ and the critical $m_{\pi}^{*}\left(M_{Q C D}\right) \sim 200$ $\mathrm{MeV}$ where the deuteron goes unbound. (Because of this fi ne-tuning, details of this fi gure depend considerably on sub-leading parameters that cannot at present be obtained from experimental data - for discussions and newer versions of Fig. 1, including unquenched lattice results, see Refs. $[20,2]$.)

The similarity with cold-atom systems under a varying magnetic fi eld is striking: QCD seems to be near a Feshbach resonance where the role of magnetic fi eld is played by the pion mass. This of course does not explain why the quark masses are such that $m_{\pi}$ is close to a quantity mostly determined by $M_{Q C D}$. But it does provide a mechanism to understand the emergence of a new momentum scale $\boldsymbol{\aleph} \sim\left|1-m_{\pi} / m_{\pi}^{*}\right| M_{\text {nис }} \ll M_{n u c}$. 


\section{Pionless EFT}

The emergence of bound states at momenta $Q \sim \aleph \ll M_{n u c}$ makes it interesting to consider an EFT where $M_{l o}=\aleph$ and $M_{h i}=M_{n u c}$. In this simpler EFT even pions and deltas can be integrated out in favor of contact interactions among nucleons; the relevant symmetries are just Lorentz and approximate parity and time-reversal. Since all that remains is contact interactions constrained by space-time symmetries, the EFT can be easily generalized [8] to atoms with large two-body scattering lengths.

Infrared enhancement is also present here, but the potential is merely a sum of delta functions and its derivatives. If the leading interactions scale with inverse powers of $\boldsymbol{\aleph}$, they need to be iterated and lead to bound states with binding momenta $Q \sim \mathcal{N}[21]$. Also like in the pionful theory, renormalization requires care because of the singular character of the interactions. In the two-body system, the delta-function strengths depend on $\Lambda$ in relatively simple ways. Corrections are treated in perturbation theory, and one can show [21] that the two-body amplitude is equivalent to the effective-range expansion. In systems with three or more bodies, things are more interesting and the virtues of EFT more evident. Some observables can be calculated to high order before new parameters appear - so-called low-energy theorems. For example, nucleon-deuteron scattering in the $S_{3 / 2}$ channel can be postdicted [22] with QED-like precision. In the $S_{1 / 2}$ channel, on the other hand, non-perturbative renormalization requires a three-body force already at leading order, which lies on a limit cycle [23] — just like two-nucleon contact interactions in the pionful theory. It has been conjectured [24] that QCD is indeed near an infrared limit cycle.

Considerable success has been achieved for bound states and scattering in systems with $A \leq 4$ nucleons $[4,6]$, which we can take as an indication that these systems are governed by the fi netuned scale $\aleph$. But nuclei get denser as $A$ increases, so it is likely that at some point binding momenta reach $M_{n u c}$. How far in $A$ can we go with this EFT? Building heavier nuclei starting from inter-nucleon interactions is in fact a major thrust of current nuclear physics. The diffi culty is that, for a given computational power, growth in $A$ can only be achieved by a reduction in number of one-particle states included in the calculation. Here, like with quarks and gluons, the numerical solution requires, in addition to an ultraviolet momentum cutoff $\Lambda$, also an infrared momentum cutoff $\lambda$ to generate a discrete one-particle basis. One such possibility is formulating [25] the EFT in a lattice of fi nite size $L$ and spacing $a$, when $\lambda \sim 1 / L$ and $\Lambda \sim 1 / a$. Another, more suitable for an eventual connection with the successful, traditional shell model, is to formulate [26] it instead within a harmonic oscillator of length $b=1 / \sqrt{m_{N} \omega}$ and a maximum number of shells $N_{\max }$, when $\lambda=1 / b$ and $\Lambda=\sqrt{\left(N_{\max }+3 / 2\right)} / b$. This method is known in nuclear physics as the No-Core Shell Model (NCSM) [27].

Using the NCSM we were able [26] to push the solution of the pionless EFT beyond $A=4$. The three leading-order parameters were fi tted at each $\Lambda$ and $\lambda$ to the deuteron, triton, and alphaparticle ground-state energies, and the energies of the ${ }^{4} \mathrm{He}$ fi rst-excited state and of the ${ }^{6} \mathrm{Li}$ ground state were postdicted. Results [26] for the ${ }^{4} \mathrm{He}$ fi rst-excited-state energy are shown in Fig. 2 for various values of $\Lambda$ and $\omega$. Convergence for $\Lambda \rightarrow \infty$ indicates correct renormalization, and the experimental value [28] is reproduced within $10 \%$ in the limit $\omega \rightarrow 0$. Results [26] for ${ }^{6} \mathrm{Li}$ are analogous, but they are about $30 \%$ off experiment. Since this is the expected size of sub-leading terms, we cannot say that we are seeing a failure of the pionless EFT. We are currently extending 

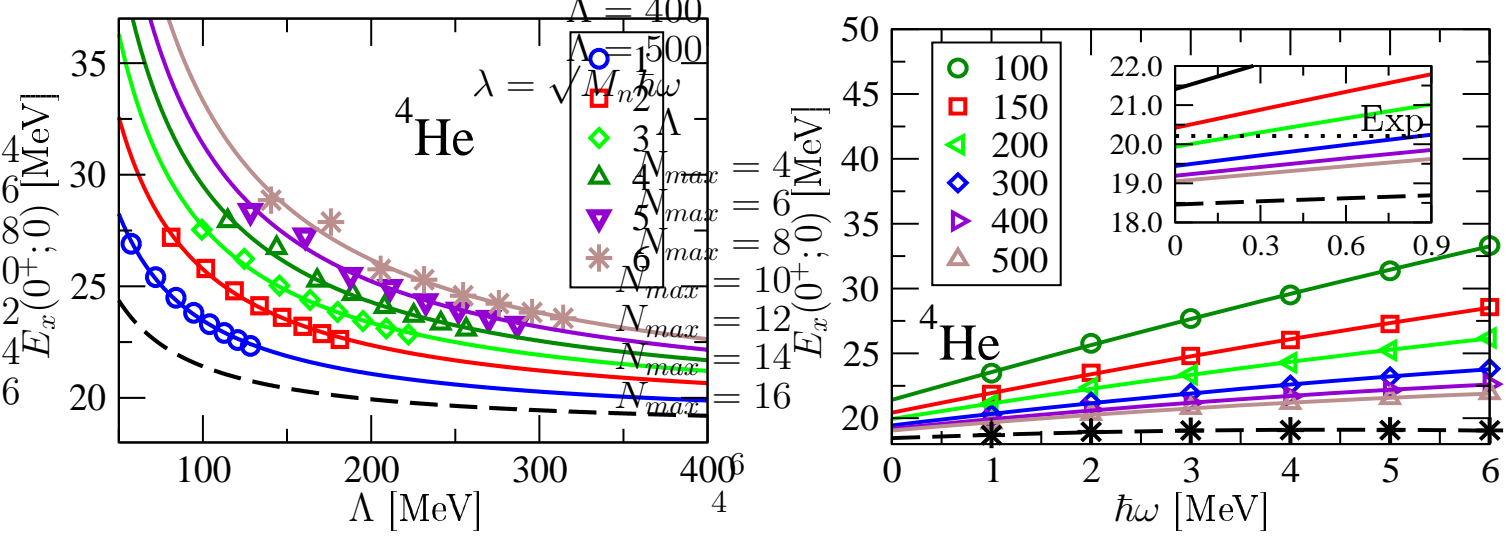

Figure 2: Dependence of the first ${ }^{4} \mathrm{He}$ excitation energy $E_{x}\left(0^{+} ; 0\right)$ on the ultraviolet momentum cutoff $\Lambda$ (left panel) and on the infrared energy cutoff ${ }^{-} h \omega$ (right panel). The points are the leading-order results [26] at the values of ${ }^{-} h \omega$ (left panel) and $\Lambda$ (right panel) given in the legends in $\mathrm{MeV}$. The (color) solid lines are simple extrapolations. The (black) dashed lines are the limits ${ }^{-} h \omega \rightarrow 0$ (left panel) and $\Lambda \rightarrow \infty$ (right panel). In the insert (right panel) we see the variation at small $h \omega \omega$, compared to the experimental value [28] given by the (black) dotted line.

the calculation to higher orders, will consider heavier nuclei, and will eventually apply the same method to the pionful EFT.

\section{Halo/Cluster EFT}

Even those powerful computational methods will run out of steam at some $A$. If we want to understand the structure of heavier nuclei we likely need to develop other EFTs. As a step in this direction, we can look at a class of nuclear systems, which are suffi ciently shallow for a cluster of nucleons to behave coherently. These are systems near thresholds for break-up into nucleons and clusters. When nucleons orbit around a core, the system is called a halo, but other cluster systems might have several cores. These systems are characterized by two scales: the energies of core excitation $E_{c}$ and of separation $E_{s} \ll E_{c}$. Classic examples involve alpha-particle cores, for which $E_{c} \sim 20 \mathrm{MeV}:{ }^{6} \mathrm{He}$, where $E_{s} \sim 1 \mathrm{MeV}$ for break-up into two nucleons and one alpha particle, and the Hoyle state of ${ }^{12} \mathrm{C}$, where $E_{s} \sim 0.3 \mathrm{MeV}$ for break-up into three alpha particles. If $\mu$ is the reduced mass, typically $\sqrt{\mu E_{s}} \sim \mathfrak{\aleph}$, while $\sqrt{\mu E_{c}} \lesssim M_{n u c}$ is a harder scale. Given that the alpha particle itself can, apparently, be described by the pionless EFT, it is not clear where this separation of scales comes from - perhaps factors of $A$.

In any case, we can formulate an EFT for such halo/cluster systems, where $M_{l o}=\sqrt{\mu E_{s}}$ and $M_{h i}=\sqrt{\mu E_{c}}$. Its structure is analogous to the pionless EFT, with the addition of a fi eld for the core. In order to determine the strengths of contact interactions in this EFT, we have at fi rst considered two-body systems: nucleon and alpha particle [29], and two alpha particles [30]. Neither system has a bound state, but they both have non-trivial low-energy physics in the form of resonances at $Q \sim \aleph$. In proton-alpha and alpha-alpha scattering the Coulomb interaction has to be incorporated explicitly. Resonances and Coulomb can be handled in EFT, with some technical developments. For reviews and references, see Refs. [6, 7]. 


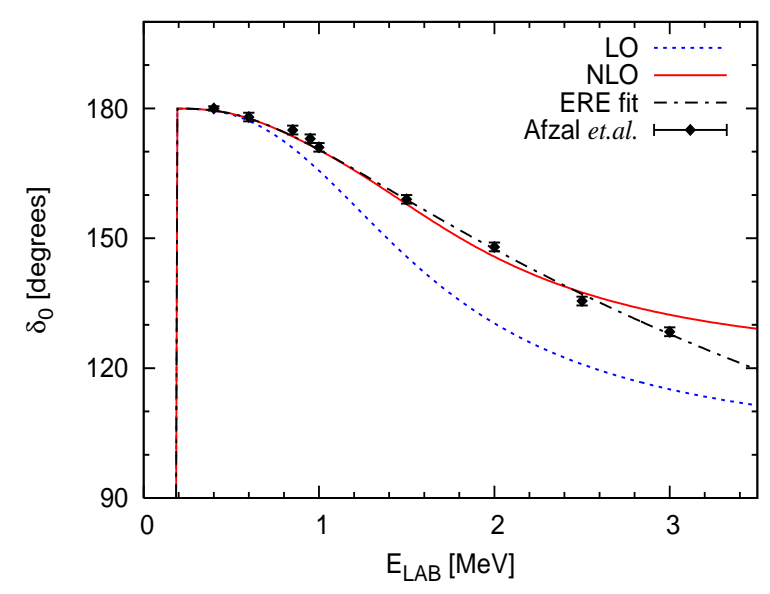

Figure 3: Two-alpha $S$-wave, Coulomb-corrected phase shift $\delta_{0}$ as a function of the laboratory energy $E_{L A B}$. The EFT results [30] in leading and next-to-leading order are given by the (blue) dotted and (red) solid lines, respectively. The empirical values [31] are the (black) solid circles with error bars, while an effective-range fit [30] is given by the (black) dash-dotted line.

In Fig. 3 I present the leading- and next-to-leading-order EFT results [30] for the two-alpha $S$ wave phase shift — with purely Coulombic interactions removed - in comparison with empirical values [31]. The rapid rise at low energy represents a resonance, which has well measured [32] energy $E_{R}=92.07 \pm 0.03 \mathrm{keV}$ and width $\Gamma\left(E_{R}\right)=5.57 \pm 0.25 \mathrm{eV}$. In leading order the EFT has two parameters, which we fi tted to these quantities; the energy dependence of the scattering amplitude is a postdiction. In next order, there is a third parameter which we fi tted to improve the energy dependence. As one can observe, a good, converging description of this system is achieved.

What is remarkable is the amount of fi ne-tuning required for such a description. The Coulomb scale for both scattering length and effective range is half the Bohr radius, about $2 \mathrm{fm}$. Coulomb indeed provides an energy dependence of the expected size; however, the strong interaction produces an energy dependence that cancels it within 10\% [30]. In order to produce a resonance at the low energy where it is observed, another factor, 100, appears and the energy-independent part of the amplitude has to have a scattering length $a_{\alpha \alpha}=-(1.92 \pm 0.09) \cdot 10^{3} \mathrm{fm} \mathrm{[30].} \mathrm{We} \mathrm{are} \mathrm{thus}$ effectively looking at a fi ne-tuning by a factor of 1000 ! Note that it is different from the one seen previously, which did not involve Coulomb. The Hoyle state of ${ }^{12} \mathrm{C}$ has long been considered [33] an example of fi ne-tuning, but the fi ne-tuning is already mind-boggling in the simpler two-alpha system. It remains to be seen if in the EFT framework the Hoyle state arises naturally from the two-alpha interactions found in the two-alpha system.

\section{Conclusion}

Many of us are prejudiced to think that fi ne-tuning does not happen. However, I have argued that at least part of the complexity found in nuclear physics is rooted in fi ne-tuning. In the process of uncovering this fi ne-tuning, quite a number of other cool features surfaced in nuclear EFTs, such as limit cycles and wide universality. 
As discussed above, there is a natural reason for nuclei to be shallow with respect to the intrinsic QCD scale — natural in the sense that it appears in the pionful EFT from the scales of spontaneous chiral symmetry breaking. Yet, the deuteron and other light nuclei are somewhat closer to being unbound than expected, which can be traced to an apparent accident: the value of the pion mass is close to a critical value. One can go a long way in explaining properties of light nuclei by incorporating this fi ne-tuning in the pionless EFT. In this context, nucleons are just cold fermions near a Feshbach resonance.

As if that was not enough, in alpha-alpha scattering in the halo/cluster EFT further fi ne-tuning seems to arise between strong and electromagnetic interactions: not one in three or four, as in the pion mass, but one in a thousand! Again the fi ne-tuning can be incorporated in the EFT, but its origin remains elusive.

EFT provides a context for emergence. And in QCD quite a lot emerges...

Acknowledgments. I thank my collaborators, particularly Renato Higa and Ionel Stetcu, for their insights into much of the work reported here.

\section{References}

[1] S. Weinberg, Phys. Lett. B251 (1990) 288; M. Rho, Phys. Rev. Lett. 66 (1991) 1275; S. Weinberg, Nucl. Phys. B363 (1991) 3; C. Ordóñez and U. van Kolck, Phys. Lett. B291 (1992) 459; S. Weinberg, Phys. Lett. B295 (1992) 114; T.-S. Park, D.-P. Min, and M. Rho, Phys. Rep. 233 (1993) 341; U. van Kolck, Phys. Rev. C 49 (1994) 2932; C. Ordóñez, L. Ray, and U. van Kolck, Phys. Rev. Lett. 72 (1994) 1982; U. van Kolck, Few-Body Syst. Suppl. 9 (1995) 444.

[2] P.F. Bedaque, talk at this conference; S.R. Beane, P.F. Bedaque, K. Orginos, and M.J. Savage, Phys. Rev. Lett. 97 (2006) 012001.

[3] W. Weise, talk at this conference.

[4] U. van Kolck, Prog. Part. Nucl. Phys. 43 (1999) 337; S.R. Beane, P.F. Bedaque, W.C. Haxton, D.R. Phillips, and M.J. Savage, in At the Frontier of Particle Physics: Handbook of QCD, edited by M.A. Shifman (World Scientific, 2001), arXiv:nucl-th/0008064; P.F. Bedaque and U. van Kolck, Ann. Rev. Nucl. Part. Sci. 52 (2002) 339.

[5] E. Epelbaum, talk at this conference; E. Epelbaum, Prog. Part. Nucl. Phys. 57 (2006) 654; U. van Kolck, Nucl. Phys. A790 (2007) 39.

[6] U. van Kolck, Nucl. Phys. A787 (2007) 405.

[7] R. Higa, arXiv:0809.5157.

[8] H.-W. Hammer, talk at this conference; E. Braaten and H.-W. Hammer, Phys. Rept. 428 (2006) 259.

[9] S. Fleming, M. Kusunoki, T. Mehen, and U. van Kolck, Phys. Rev. D 76 (2007) 034006; S. Fleming and T. Mehen, arXiv:0807.2674 [hep-ph].

[10] A. Nogga, R.G.E. Timmermans, and U. van Kolck, Phys. Rev. C 72 (2005) 054006; M.C. Birse, Phys. Rev. C 74 (2006) 014003; M. Pavón Valderrama and E. Ruiz Arriola, Phys. Rev. C 74 (2006) 064004; M.C. Birse, Phys. Rev. C 76 (2007) 034002.

[11] J.L. Friar, D. Hüber, and U. van Kolck, Phys. Rev. C 59 (1999) 53; D. Hüber, J.L. Friar, A. Nogga, H. Witała, and U. van Kolck, Few-Body Syst. 30 (2001) 95; S.A. Coon and H.K. Han, Few-Body Syst. 30 (2001) 131. 
[12] M.C.M. Rentmeester, R.G.E. Timmermans, J.L. Friar, and J.J. de Swart, Phys. Rev. Lett. 82 (1999) 4992; M.C.M. Rentmeester, R.G.E. Timmermans, and J. J. de Swart, Phys. Rev. C 67 (2003) 044001.

[13] A. Manohar and H. Georgi, Nucl. Phys. B234 (1984) 189.

[14] S.R. Beane, P.F. Bedaque, L. Childress, A. Kryjevski, J. McGuire, and U. van Kolck, Phys. Rev. A 64 (2001) 042103; M. Bawin and S.A. Coon, Phys. Rev. A 67 (2003) 042712; E. Braaten and D. Phillips, arXiv:hep-th/0403168; M. Alberg, M. Bawin, and F. Brau, Phys. Rev. A 71 (2005) 022108; H.E. Camblong and C.R. Ordóñez, Phys. Lett. A345 (2005) 22; H.-W. Hammer and B.G. Swingle, Ann. Phys. 321 (2006) 306.

[15] B. Long and U. van Kolck, Ann. Phys. 323 (2008) 1304.

[16] D.B. Kaplan, M.J. Savage, and M.B. Wise, Nucl. Phys. B478 (1996) 629.

[17] S.R. Beane, P.F. Bedaque, M.J. Savage, and U. van Kolck, Nucl. Phys. A700 (2002) 377.

[18] E. Epelbaum and U.-G. Meißner, arXiv:nucl-th/0609037.

[19] M. Fukugita, Y. Kuramashi, M. Okawa, H. Mino, and A. Ukawa, Phys. Rev. D 52 (1995) 3003.

[20] S.R. Beane and M.J. Savage, Nucl. Phys. A713 (2003) 148; E. Epelbaum, U.-G. Meißner, and W. Glöckle, Nucl. Phys. A714 (2003) 535; S.R. Beane and M.J. Savage, Nucl. Phys. A717 (2003) 91; E. Epelbaum, U.-G. Meißner, and W. Glöckle, arXiv:nucl-th/0208040.

[21] U. van Kolck, in Workshop on Chiral Dynamics 1997, Theory and Experiment, edited by A. Bernstein, D. Drechsel, and T. Walcher (Springer-Verlag, 1997), arXiv:hep-ph/9711222; D.B. Kaplan, M.J. Savage, and M.B. Wise, Phys. Lett. B424 (1998) 390; J. Gegelia, in Nonperturbative Methods in Quantum Field Theory, edited by A.W. Schreiber, A.G. Williams, and A.W. Thomas (World Scientific, 1998), arXiv:nucl-th/9802038; U. van Kolck, Nucl. Phys. A645 (1999) 273.

[22] P.F. Bedaque and U. van Kolck, Phys. Lett. B428 (1998) 221; P.F. Bedaque, H.-W. Hammer, and U. van Kolck, Phys. Rev. C 58 (1998) R641; F. Gabbiani, P.F. Bedaque, and H.W. Grießhammer, Nucl. Phys. $\mathbf{A 6 7 5}$ (2000) 601; M.C. Birse, arXiv:nucl-th/0509031.

[23] P.F. Bedaque, H.-W. Hammer, and U. van Kolck, Nucl. Phys. A676 (2000) 357; H.-W. Hammer and T. Mehen, Phys. Lett. B516 (2001) 353; P.F. Bedaque, G. Rupak, H.W. Grießhammer, and H.-W. Hammer, Nucl. Phys. A714 (2003) 589; H.W. Grießhammer, Nucl. Phys. A744 (2004) 192; I.R. Afnan and D.R. Phillips, Phys. Rev. C 69 (2004) 034010; L. Platter, Phys. Rev. C 74 (2006) 037001.

[24] E. Braaten and H.-W. Hammer, Phys. Rev. Lett. 91 (2003) 102002; E. Epelbaum, H.-W. Hammer, U.-G. Meißner, and A. Nogga, Eur. Phys. J. C48 (2006) 169; H.-W. Hammer, D.R. Phillips, and L. Platter, Eur. Phys. J. A32 (2007) 335.

[25] D. Lee, talk at this conference; D. Lee, arXiv:0804.3501.

[26] I. Stetcu, B.R. Barrett, and U. van Kolck, Phys. Lett. B653 (2007) 358.

[27] P. Navrátil, J.P. Vary, and B.R. Barrett, Phys. Rev. Lett. 84 (2000) 5728; Phys. Rev. C 62 (2000) 054311.

[28] D.R. Tilley, H.R. Weller, and G.M. Hale, Nucl. Phys. A541 (1992) 1.

[29] C.A. Bertulani, H.-W. Hammer, and U. van Kolck, Nucl. Phys. A712 (2002) 37; P.F. Bedaque, H.-W. Hammer, and U. van Kolck, Phys. Lett. B569 (2003) 159; C.A. Bertulani, R. Higa, and U. van Kolck, in preparation.

[30] R. Higa, H.-W. Hammer, and U. van Kolck, Nucl. Phys. A809 (2008) 171. 
[31] S.A. Afzal, A.A.Z. Ahmad, and S. Ali, Rev. Mod. Phys. 41 (1969) 247.

[32] S. Wüstenbecker, H.W. Becker, H. Ebbing, W.H. Schulte, M. Berheide, M. Buschmann, C. Rolfs, G.E. Mitchell, and J.S. Schweitzer, Z. Phys. A344 (1992) 205.

[33] H. Oberhummer, H. Krauss, K. Grün, T. Rauscher, H. Abele, P. Mohr, and G. Staudt, arXiv:astro-ph/9310026; H. Schlattl, A. Heger, H. Oberhummer, T. Rauscher, and A. Csótó, Astrophys. Space Sci. 291 (2004) 27. 